Conformal dip-coating of patterned surfaces for capillary die-to-substrate self-assembly

This article has been downloaded from IOPscience. Please scroll down to see the full text article.

2009 J. Micromech. Microeng. 19045015

(http://iopscience.iop.org/0960-1317/19/4/045015)

View the table of contents for this issue, or go to the journal homepage for more

Download details:

IP Address: 128.178.244.221

The article was downloaded on 09/11/2010 at 17:27

Please note that terms and conditions apply. 


\title{
Conformal dip-coating of patterned surfaces for capillary die-to-substrate self-assembly
}

\author{
M Mastrangeli ${ }^{1,2}$, W Ruythooren ${ }^{1}$, C Van Hoof ${ }^{1}$ and J-P Celis ${ }^{2}$ \\ ${ }^{1}$ IMEC, Kapeldreef 75, 3001 Leuven, Belgium \\ 2 Department MTM, Katholieke Universiteit Leuven, Kasteelpark Arenberg 44, 3001 Leuven, Belgium \\ E-mail: mastran@imec.be
}

Received 5 November 2008, in final form 16 January 2009

Published 17 March 2009

Online at stacks.iop.org/JMM/19/045015

\begin{abstract}
Capillarity-driven self-assembly of small chips onto planar target substrates is a promising alternative to robotic pick-and-place assembly. It critically relies on the selective deposition of thin fluid films on patterned binding sites, which is anyway normally non-conformal. We found that the addition of a thin wetting sidewall, surrounding the entire site perimeter, enables the conformal fluid coverage of arbitrarily shaped sites through dip-coating, significantly improves the reproducibility of the coating process and strongly reduces its sensitivity to surface defects. In this paper we support the feasibility and potential of this method by demonstrating the conformal dip-coating of square and triangular sites conditioned with combinations of different hydrophobic and hydrophilic surface chemistries. We present both experimental and simulative evidence of the advantages brought by the introduction of the wetting boundary on film coverage accuracy. Application of our surface preparation method to capillary self-assembly could result in higher precision in die-to-substrate registration and larger freedom in site shape design.
\end{abstract}

(Some figures in this article are in colour only in the electronic version)

\section{Introduction}

During the last two decades, self-assembly [1-3] emerged as a promising alternative to robotic pick-and-place $[4,5]$ for the assembly of microsystems [5, 6]. Self-assembly combines non-prehensile manipulation of components, which avoids stiction issues [5, 7, 8], with stochastic assembly principles to enable handling parallelism. Several embodiments of this manufacturing paradigm were proposed based on different actuation principles, such as fluidic and gravitational [9-12], electric field-mediated [13-16], magnetic [17-19], shapedirected [20], molten-solder-driven [21, 22] and capillary ones $[23,24]$. These approaches proved successful in assembling a variety of structures and devices, including LEDs [9, 13, 24], lasers [25], three-dimensional electrical networks [26], cylindrical displays [21, 27], micromirrors [28], resistors [29], inductors [30], actuators [31], HEMTs [32] and single-crystal MOS transistors [22].
Particularly, capillary self-assembly [33] shows promise for massively parallel handling of components and for high accuracy in component alignment and registration [23]. This approach combines transport and dispensing of components in a fluid environment with the stochastic attachment of the components to properly coated binding sites patterned on a planar template substrate [27]. It critically relies on the selective and conformal deposition of thin fluid films over surfaces structured with areas of contrasting wetting properties.

The simplest technique used to coat large arrays of patches on planar substrates is dip-coating [34]. This technique consists in sliding the substrate at a controlled velocity across the interface between a wetting fluid and a non-wetting one. Extensive literature on the dip-coating of planar substrates is available [35-39]. Nonetheless, to our knowledge only limited work was dedicated so far to the investigation of conformal dip-coating of arrays of patches patterned on planar substrates. 


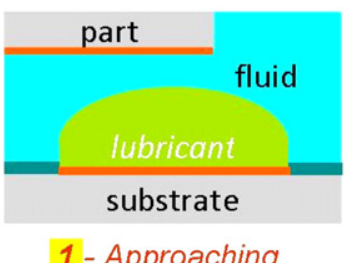

1 - Approaching

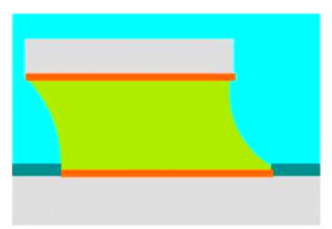

2 - Energy minimization

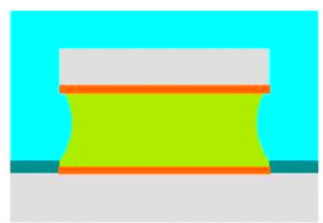

3 - Self-alignment

Figure 1. Dynamics of capillary self-assembly. Step 1: a part, suspended in a hosting fluid, approaches a binding site, selectively coated with a thin fluid (i.e. lubricant) film whose interface with the immiscible hosting fluid has high energy. Step 2: as the part touches the lubricant surface, the former hosting fluid/lubricant interface is substituted with the less energetic part/lubricant one. Step 3: the force deriving from interfacial energy minimization (i.e. capillarity) drives the part into alignment with the site underneath.

In this paper, an experimental and simulative study on the conformality of the dip-coating of chemically and topologically patterned surfaces is presented. After describing the capillary self-assembly process and the importance of the fluid coverage of the binding sites for its yield, we define the topological approach we adopted to obtain the conformal coverage of the sites. For this purpose, we compare the geometry of superficial sites and of recessed sites, i.e. of sites without and with surrounding wetting sidewall, respectively. We then show experimental evidence concerning the nonconformality of the coverage that is generally achieved over substrates patterned with superficial sites having polygonal shapes with non-curvilinear perimeters. This is compared to the perfectly conformal coverage that can be obtained using recessed sites of the same shapes. Subsequently, we support our experimental observations with finite element simulations.

\section{Capillary die-to-substrate self-assembly}

\subsection{The process}

The capillary die-to-substrate assembly technique is schematically depicted in figure 1 . The target substrate is immersed in the hosting fluid (normally, a water-based solution) and is patterned with arbitrary arrays of hydrophobic binding sites separated by hydrophilic spacer areas. The binding sites are selectively covered with lenses of a hydrophobic lubricant (e.g. a hydrocarbon- or fluorocarbonbased polymer). The lubricant must wet the binding sites. It is typically selected so that its interface with the hosting fluid is highly energetic. The components to be assembled onto the binding sites are in turn chemically treated to make their functional side (i.e. the side exposing the electrical or functional connections to be bound to the substrate) hydrophobic, while leaving the other sides hydrophilic. During assembly, the components are spread and stirred into the hosting fluid, where they wander across the substrate. Upon stochastic contact between the lubricant lenses and the hydrophobic side of the components, the energy of the system decreases, since the highly energetic lubricant/hosting fluid interface is substituted by a less energetic one. This event produces a force, both lateral and vertical, which drives the components to maximize their overlap with the underlying binding sites, i.e. to minimize the area of the lubricant menisci.
Given a properly matching design of both binding sites and components, such capillary actuation drives the components to their final equilibrium position. In the set-up described above, the selective affinity of the components to the binding sites is mediated by the hydrophobic interaction [40]. Using a hydrophobic fluid (such as air [41, 42]) as hosting medium, the assembly concept remains the same but the wetting properties of binding sites and spacer areas must be inverted.

\subsection{Importance of conformal site coverage}

The yield of capillary self-assembly depends on several parameters, among which are the wetting properties of various surfaces, and most importantly the coverage of the binding sites with the hydrophobic lubricant. The lubricant has multiple functions: (1) it largely amplifies the spatial range where the hydrophobic interaction, due to the hydrophobic binding sites, is effective; (2) in combination with the hosting liquid, it provides the interfacial energy which is the source of the capillary restoring forces on the components; and (3) it avoids friction between the surfaces of the components and the substrate, thus allowing the components to explore their assembly space to reach their energetically optimal positions [43]. Given the geometrical congruence (within lithographic tolerances) of the mating surfaces, the precision of the alignment between the component to be assembled and the underlying binding site is expected to depend, apart from the properties of the lubricant and accidental surface defects, on the conformality of the fluid lenses to the pattern of the site. Indeed, upon approach towards the binding sites, what the components actually find and interact with is the lubricant. Thus, the lubricant needs to replicate the exact profile of the site with high fidelity, in order to convey the exact information required for the self-alignment. This is particularly relevant when patterned details are purposely added to the site design to determine or enhance the alignment and orientation of the components [44, 45]. Sato et al confirmed experimentally that wetting defects and poor conformality of the fluid lenses to the binding sites directly translate into misalignment of components assembled by capillarity [46]. The worst performance was achieved with shapes featuring sharp corners or convexities, such as crosses, stars and, above all, triangles. 


\section{Superficial versus recessed binding sites}

A chemical heterogeneity of the surface of a planar substrate can be exploited to direct the selective coating of this surface with two immiscible fluids. For this purpose, the substrate needs to be patterned with adjacent regions of contrasting wetting properties, such as hydrophobic and hydrophilic ones. When the fluids get in contact with the surface, they organize and segregate into three-dimensional structures reflecting the wettability and the geometrical patterns of the regions.

In the dip-coating technique, the immiscible fluids are sequentially brought into contact with the pre-patterned surface by sliding the entire substrate through their interface. In a common set-up, a hydrocarbon floats on top of water in a container. The substrate to be coated is vertically lowered through the horizontal hydrocarbon/water interface, and allowed to rest in water. As a result of minimization of interfacial energy, the hydrocarbon is repelled from the hydrophilic regions, which are instead wet by water, and self-assembles into lenses on the hydrophobic regions [47]. The rate at which the substrate is immersed, together with the fluid interfacial tension, the fluid viscosity, and the size and geometry of the sites, affects the shapes and volume of the resulting fluid structures, which in turn have direct consequences on the self-assembly dynamics [48, 49].

The shape of a fluid lens chemically confined inside a planar superficial patch (figure $2(a)$ ) is affected by the fluid volume, its surface tension, its chemical affinity to the surface underneath and by the shape of the confining patch [50]. Particularly, for polygonal shapes, incomplete wetting of sharp angles was reported [51]. Such partial coverage may be reduced by decreasing the interfacial tension $\gamma_{\mathrm{LH}}$ between the lubricant and the hosting fluid. This would increase the wetting capability of the lubricant by decreasing its contact angle $\theta$ (defined according to the Young-Laplace equation as $\cos \theta \equiv\left(\gamma_{\mathrm{SH}}-\gamma_{\mathrm{SL}}\right) / \gamma_{\mathrm{LH}}$, where $\gamma_{\mathrm{SH}}$ and $\gamma_{\mathrm{SL}}$ represent the interfacial tension between the solid substrate and the hosting fluid and the solid substrate and the lubricant, respectively) and equivalently increasing its spreading coefficient $S \equiv$ $\gamma_{\mathrm{SH}}-\gamma_{\mathrm{SL}}-\gamma_{\mathrm{LH}}$. However, this would at the same time decrease the magnitude of the shear capillary forces inducing and restoring the alignment of the assembling component, because these forces are proportional to $\gamma_{\mathrm{LH}}$ [49]. This would make the assembly more susceptible to misalignments and external perturbations.

To achieve a perfectly conformal coverage of arbitrary sites with lubricant while preserving the lubricant properties, we acted on the site geometry. We introduced a relatively thin wetting sidewall around the entire perimeter of the binding site. The pattern of the site was otherwise not modified; i.e. the site was recessed into a shallow cavity (figure $2(b)$ ). The additional contour was made chemically homogeneous to the rest of the recessed site. It thus pulled the lubricant towards the edges of the site, thereby enforcing pinning and the complete, conformal coverage of the entire site.

In the perspective of capillary assembly, circular binding sites do not induce any in-plane orientation, as all possible orientations of the assembled components are energetically (a) Superficial site

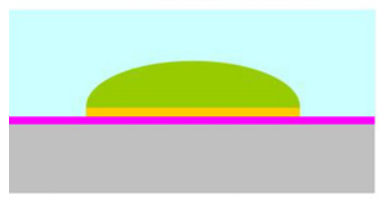

Lubricant film

Substrate Hosting fluid (b) Recessed site

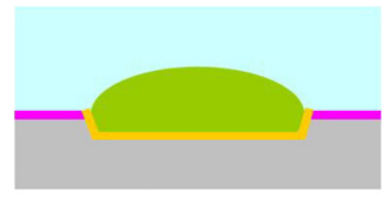

Hydrophilic layer

Hydrophobic layer
Figure 2. Schematic cross-section of superficial $(a)$ and recessed (b) planar binding sites. Relative dimensions are out of scale.

equivalent. While the search for specific site design that can induce unique in-plane orientation is still ongoing [44, 45], the most common, and easy to fabricate, shapes adopted so far in capillary assembly are squares and rectangles (which have intrinsic fourfold and twofold symmetry, respectively) and, more generally, polygons. In the following sections, we investigate the thin film coverage of polygonally-patterned, chemically-treated binding sites on planar substrates, as obtained by a dip-coating process.

\section{Materials and methods}

\subsection{Samples preparation}

To demonstrate the effect of the sidewall on site coverage, we first focused on square binding sites with side length $L=1 \mathrm{~mm}$. We fabricated both superficial and recessed binding sites using three combinations of surface chemistries to define their hydrophobic and hydrophilic regions, for a total of six types of sites (sketched in figure 3). In all cases, we started with (1 $\left.\begin{array}{lll}0 & 0\end{array}\right)$ silicon substrates with a $250 \mathrm{~nm}$ thick thermal silica layer as the top surface. We patterned $10 \times 10$ arrays of sites and we diced the substrates into $2.2 \times 2.2 \mathrm{~cm}^{2}$ chips for dipcoating experiments. The first class of patterns, referred to as $\mathrm{CH}_{3} / \mathrm{SiO}_{2}$ in the following, had hydrophobic binding sites made of methyl groups separated by hydrophilic silica spacer areas. The second class of patterns, referred to as $\mathrm{Si}-\mathrm{H} / \mathrm{SiO}_{2}$, had hydrophobic binding sites made of hydrogen-terminated silicon separated by hydrophilic silica spacer areas. The third class of patterns, referred to as $\mathrm{SiO}_{2} / \mathrm{CH}_{3}$, had hydrophilic binding sites made of silica separated by hydrophobic, methylterminated spacer areas.

To realize superficial sites of the first class, a Ti/Au metal layer (50/100 nm thick, respectively) was evaporated over the silica surface and lithographically patterned into squares. For the recessed sites of the same class, $2.5 \mu \mathrm{m}$ deep cavities were etched into the silicon substrate by $\mathrm{SF}_{6} /$ Ar-based reactive ion etching (RIE). The Ti/Au metal layer was then evaporated and patterned by resist lift-off. The superficial site had the metal layer on top of the silica surface, devoid of any peripheral wetting layer; in the recessed type, the metal layer was located below the silica surface. After dicing, the chips 


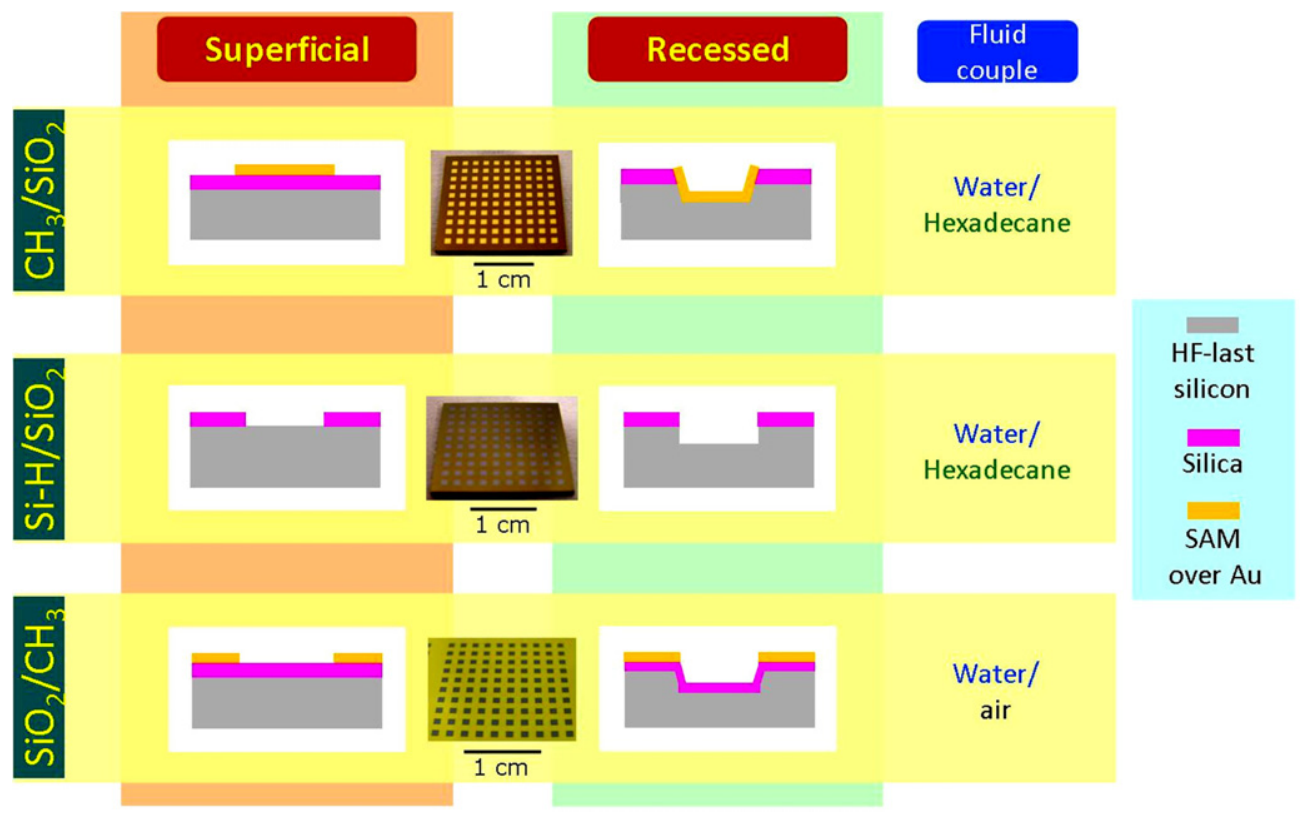

Figure 3. The six types of square sites used in this work. Relative dimensions of the layers in the sketches are out of scale for illustration purposes.

were thoroughly rinsed with acetone, isopropyl alcohol and de-ionized (DI) water, soaked in $30 \%$ hydrogen peroxide for $15 \mathrm{~min}$, dried under a nitrogen flow and finally stored overnight in a $1 \mathrm{mM}$ solution of dodecanethiol $\left(\mathrm{CH}_{3}\left(\mathrm{CH}_{2}\right)_{11} \mathrm{SH}\right)$ in pure ethanol. As a result of this last step, self-assembled monolayers (SAMs) [52] selectively bound to the Au sites with their thiol headgroups, while exposing their methyl endgroups at the surface. Just before dip-coating, the chips were extracted from the SAM solution, rinsed with ethanol to remove excess unbound organic molecules and dried with nitrogen.

Superficial sites of the second class were realized by etching away the silica layer in diluted hydrofluoric acid (HF), exposing the underlying silicon substrate in square regions. Recessed sites of the same class were obtained simply by adding, after $\mathrm{SiO}_{2}$ etch, the same $2.5 \mu \mathrm{m}$ deep RIE step as above to create shallow cavities inside silicon. In this class, both types of sites lay below the silica surface. They both had peripheral walls. However, in the superficial type the walls were not chemically homogeneous with the rest of the site, being entirely made of silica, while in the recessed type their largest part was constituted of silicon. Immediately before dip-coating, the chips were immersed in ammonium fluoride for $5 \mathrm{~s}$, then rinsed with DI water and dried with nitrogen. This was done to passivate the bare silicon surface of the sites with hydrogen, thus making it hydrophobic [53], while preserving the hydrophilic silica spacer all around.

To fabricate superficial sites of the third class, the $\mathrm{Ti} / \mathrm{Au}$ metal layer was evaporated on the silica surface and lithographically patterned to expose silica in square areas. For the recessed sites, the metal layer was used as hard mask during a subsequent $\mathrm{SiO}_{2}$ etch in diluted $\mathrm{HF}$ and an anisotropic silicon etch in potassium hydroxide to create $5 \mu \mathrm{m}$ deep sloped cavities. The bare silicon surface was in this case not passivated to keep it hydrophilic. Dodecanethiol SAMs were then assembled on the exposed Au surface, using the same procedure described above, to make the spacer areas hydrophobic. Also in this class both types of sites lay below the top surface level, but only the recessed type had a uniformly defined wetting sidewall around the site perimeter.

Water contact angles (CAs) were measured on dummy chips to test the conditions of the chemically treated surfaces after complete sample preparation and before dip-coating. The CAs were measured with an OCA-20 contact angle meter (DataPhysics Instruments $\mathrm{GmbH}$ ) with SCA20 software, using $1 \mu$ l water drops.

\section{2. dip-coating procedures}

All dip-coating experiments were performed at room temperature. We chose water and hexadecane (HD) as immiscible liquids characterized by a high interfacial energy $\left(\gamma_{\mathrm{HD} / \mathrm{H}_{2} \mathrm{O}} \cong 53 \mathrm{~mJ} \mathrm{~m}^{-2}\right)$ [51]. As the capillary length for this fluid couple $L_{c}^{\mathrm{HD} / \mathrm{H}_{2} \mathrm{O}}=\sqrt{\gamma_{\mathrm{HD} / \mathrm{H}_{2} \mathrm{O}} / g \Delta \rho}=4.88 \mathrm{~mm}$ was larger than the linear site dimension, and the Bond number $B o^{\mathrm{HD} / \mathrm{H}_{2} \mathrm{O}}=\Delta \rho g L^{2} / \gamma_{\mathrm{HD} / \mathrm{H}_{2} \mathrm{O}}=0.04 \ll 1$, gravitational effects were negligible compared to surface tension effects on the formation of fluid lenses $\left(\Delta \rho=\rho_{\mathrm{H}_{2} \mathrm{O}}-\rho_{\mathrm{HD}}\right)$.

In order to cover with lubricant the first two classes of sites, we adopted a dip-coating procedure analogous to that presented in [47]. The experimental set-up is shown in figure 4. A PTFE holder, featuring square, shallow compartments, roughly of the size of the chips, was placed at the base of a DI water-filled beaker, and a Pyrex tube was inserted right above the holder to confine a $1 \mathrm{~mm}$ thick HD film, floating on the water surface. After complete preparation, the samples were individually, manually and vertically lowered inside the tube across the $\mathrm{HD}$ /water interface, with the rows of sites parallel to the liquid interfaces, and then left lying in 


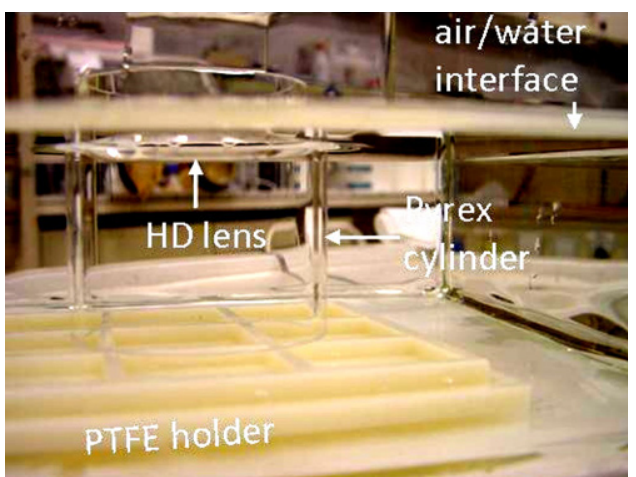

Figure 4. Experimental dip-coating set-up. The PTFE holder stands on the bottom of the water-filled beaker. The Pyrex cylinder confines the HD lens, which floats on water. The substrates patterned with binding sites will be slid through the air/HD/water interface inside the cylinder.

the holder compartments in water. The lowering speed of the substrate was kept to around $1 \mathrm{~mm} \mathrm{~s}^{-1}$; the corresponding capillary number was $\mathrm{Ca}=\frac{\mu U}{\gamma_{\mathrm{HD} / \mathrm{H}_{2} \mathrm{O}}}=5.7 \times 10^{-5}, \mu$ being the lubricant viscosity and $U$ the dip-coating speed. After the experiments, the tube was removed away from above the holder, so that the holder could be taken out from the beaker through a clean water/air interface. By keeping the holder horizontal and parallel to the interface, enough water remained in the compartments so as to continuously submerge the chips, also while transporting the holder in ambient air. Optical photographs of the chips, resting in water on the holder, were taken to analyse the results of the coating experiments.

For the third class of sites, water and air were chosen as the immiscible fluid couple $\left(\gamma_{\mathrm{H}_{2} \mathrm{O}}=72.8 \mathrm{mJm}^{-2}, L_{c}^{\mathrm{H}_{2} \mathrm{O}}=\right.$ $2.72 \mathrm{~mm}, \mathrm{Bo}^{\mathrm{H}_{2} \mathrm{O}}=0.13$ ) to prove that the results were independent of the specific fluid couple used. The chips were immersed in a DI water-filled beaker and vertically, manually withdrawn, keeping the rows of binding sites parallel to the water/air interface, at a speed of about $1 \mathrm{~mm} \mathrm{~s}^{-1}$ $\left(C a=1.37 \times 10^{-5}\right)$. Since the laboratory atmosphere was not saturated with water vapour, less than a minute after extraction from the beaker evaporation severely perturbed the shape of the water lenses till their complete disappearance. Optical pictures of the lenses were captured well before this happened.

\section{Experimental results}

The average CAs of water measured on thermal silica and bare silicon surfaces were below $10^{\circ}$, which is the lowest CA value precisely measurable by our instrument. In contrast, CAs on dodecanethiol-functionalized Au surfaces and on hydrogenated Si surfaces were close to $110^{\circ}$ and above $70^{\circ}$, respectively. This proves that the conditioning was properly done, since the treated surfaces had a strong chemical heterogeneity.

\subsection{First class of sites: $\mathrm{CH}_{3} / \mathrm{SiO}_{2}$}

Experimental results for dip-coated superficial sites of the $\mathrm{CH}_{3} / \mathrm{SiO}_{2}$ class revealed that, while the process appeared to

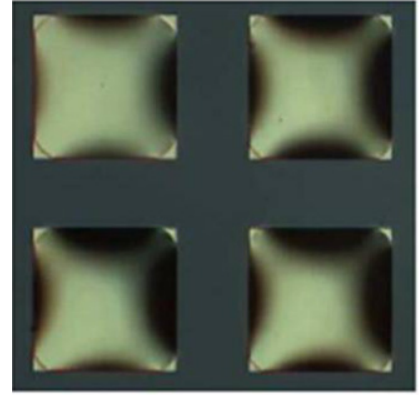

(a)

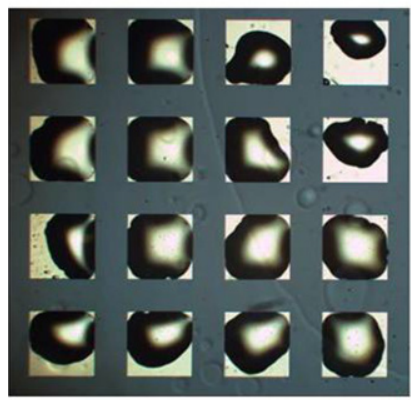

(c)

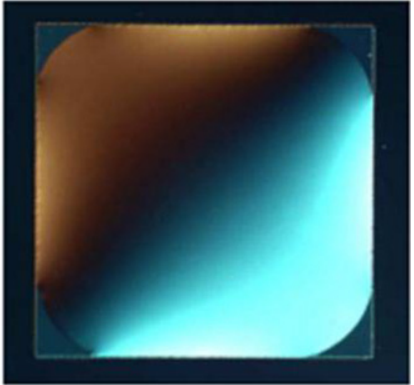

(b)

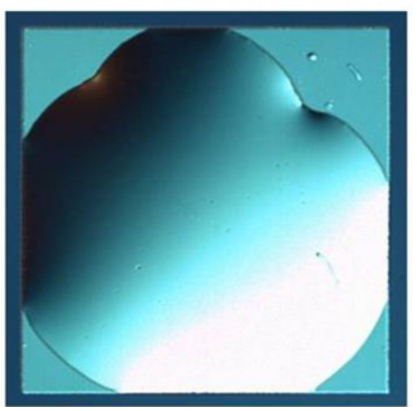

(d)
Figure 5. Hexadecane dip-coating of superficial square $\mathrm{CH}_{3} / \mathrm{SiO}_{2}$ sites. (a) Typical dip-coating result. (b) Close-up evidencing incomplete coverage of corners. $(c)$ Absence of lens pinning enables occasional strong perturbations of lens shapes as resulting from various surface defects. $(d)$ Close-up evidencing the variable position of the triple-contact line. The substrates are submerged in water. Colours and depth enhanced by differential interference contrast (DIC) optical microscopy. All site sides are $1 \mathrm{~mm}$ long.

be repeatable and oil lenses followed the in-plane rotational symmetry of the sites, complete site coverage was never achieved (figure 5). Indeed the lubricant film appeared to be constrained by the side edges of the sites, but it was not pinned by them and it did not completely reach into the corners. The absence of pinning, especially in the corners, allowed for fluctuations of the position of the triple-contact line, because of its susceptibility to local surface defects (e.g. scratches, particles, resist residues, incomplete SAM formation on $\mathrm{Au}$ surfaces) and to variations in the local dynamics of the coating process (figures $5(a)$ and $(b)$ ). Such fluctuations implied a site-to-site difference in the relative surface coverage and most probably of the volume of the lubricant lenses, even for sites in close proximity. The process reproducibility was consequently affected. These results were consistent with previous reports [23].

Evident benefits came from adding a wetting, hydrophobic sidewall to the sides. Recessed $\mathrm{CH}_{3} / \mathrm{SiO}_{2}$ square sites were routinely dip-coated showing complete lubricant adhesion to the entire perimeter of the pattern, including all corners (figures $6(a)$ and $(b)$ ). More than $98 \%$ of the processed sites were correctly covered, indicating a superior robustness against surface defects (figures 6(c) and $(d)$ ). Minimal dewetting was only rarely seen in random locations, always close to the site edges, hinting at local pinning of the HD lenses 


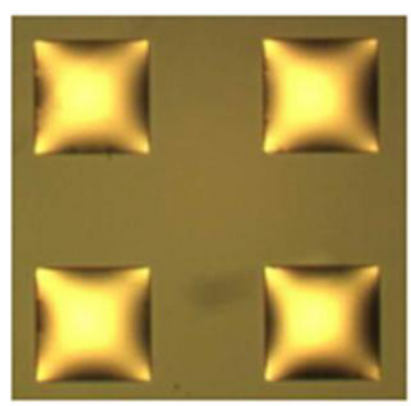

(a)

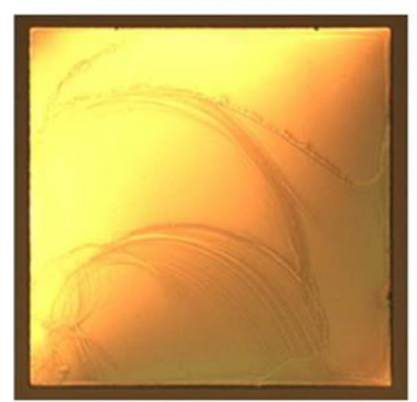

$(c)$

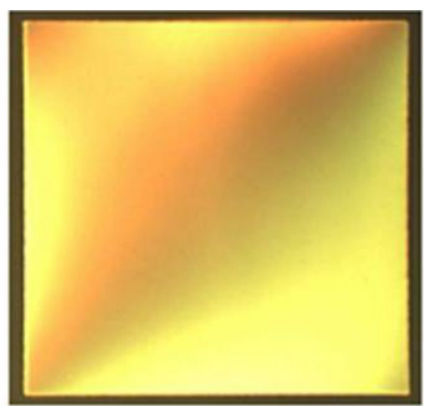

(b)

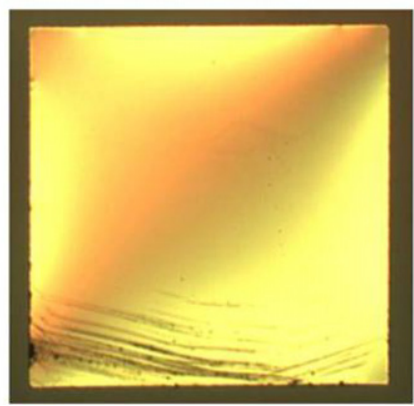

(d)

Figure 6. Hexadecane dip-coating of recessed square $\mathrm{CH}_{3} / \mathrm{SiO}_{2}$ sites. Perfectly conformal coating of sites is routinely achieved $(a)$; complete corners wetting is evidenced in the close-up $(b)$. The coating is robust against imperfect surface cleaning $(c)$ and surface scratches $(d)$. The substrates are submerged in water. Colours and depth enhanced by DIC optical microscopy. All site sides are $1 \mathrm{~mm}$ long.

due to imperfections in the lift-off process as the possible cause.

\subsection{Second class of sites: $\mathrm{Si}-\mathrm{H} / \mathrm{SiO}_{2}$}

A similar scenario appeared comparing dip-coating results for sites of the $\mathrm{Si}-\mathrm{H} / \mathrm{SiO}_{2}$ class. Covering this class of superficial sites was almost impossible. Most sites had no significant lubricant coverage at all. The few covered sites were always metastable, quickly experiencing capillary lens break-up after HD deposition. A partial explanation may involve the hydrophilicity of the entirety of the shallow $\mathrm{SiO}_{2}$ sidewalls which, being wetted by water, prevented any contact with the hydrocarbon and thus its pinning.

Conversely, recessed $\mathrm{Si}-\mathrm{H} / \mathrm{SiO}_{2}$ sites revealed a coverage performance analogous to that of the first recessed class (figures $7(a)$ and $(b)$ ). While perfect fluid film conformality was typically found, the coverage yield was lower than for the first recessed class, due to a higher incidence of local surface imperfections, such as e.g. incomplete Si passivation. For these cases, large de-wetted areas were seen, together with asymmetric HD drops (figure $7(d)$ ). These drops were always clamped to a fraction of one or more edges of the cavities, probably resulting from the capillary break-up of former full site-covering lenses.

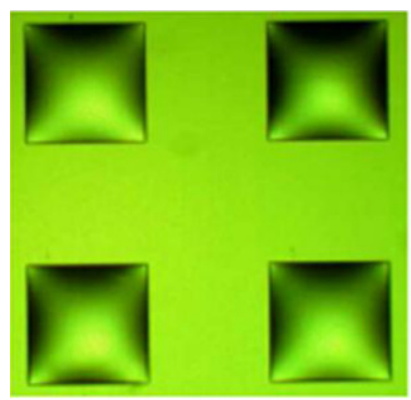

(a)

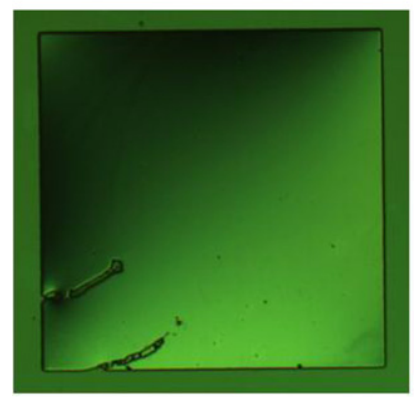

(c)

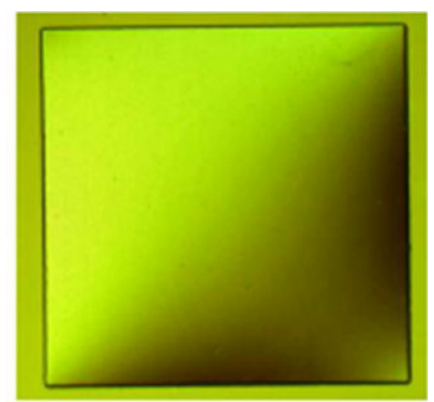

(b)

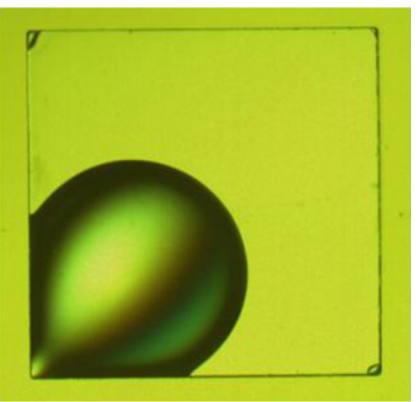

(d)
Figure 7. Hexadecane dip-coating of recessed $\mathrm{Si}-\mathrm{H} / \mathrm{SiO}_{2}$ square sites. Complete coating $(a)$, emphasized in the close-up $(b)$, is normally achieved. (c) Robustness against surface defects.

(d) Asymmetric lens pinned along the edges as resulting from capillary break-up of a former full site-covering lens. The substrates are submerged in water. Colours and depth enhanced by DIC optical microscopy. All site sides are $1 \mathrm{~mm}$ long.

\subsection{Third class of sites: $\mathrm{SiO}_{2} / \mathrm{CH}_{3}$}

The results for the third class of samples followed the same trend as those for the first class. Non-conformal coverage was seen in most cases for superficial sites (figure $8(a)$ ). This observation is interesting because (1) the topology of the sites is similar, except for the absence of a wetting sidewall around the site, to that of the wells studied in [39] for which conformal filling was reported by so-called discontinuous de-wetting; (2) the small CA of water on silica should enforce the complete wetting of the surface. As expected, dip-coating of recessed $\mathrm{SiO}_{2} / \mathrm{CH}_{3}$ sites produced perfectly conformal fluid coverage of the sites (figure $8(b)$ ). Interestingly, the two different site topologies resulted in different water evaporation patterns. For superficial sites, evaporation produced lens shrinking which started from the lens edges (figure 8(c)), resembling a constant contact angle drop evaporation modality [54]. In contrast, in recessed sites lens break-up caused by evaporation started from the centre and moved towards the site edges (figure $8(d)$ ), confirming the strong fluid pinning at the site sidewalls.

\subsection{Triangular sites}

Further, we wanted to confirm that with our method even polygons with extremely acute angles and very anisotropic shapes could be conformally covered with lubricant, and that the performance was rather scale independent. For this 


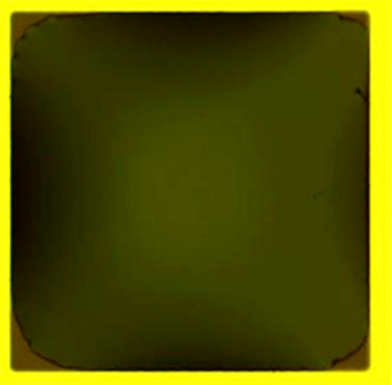

(a)

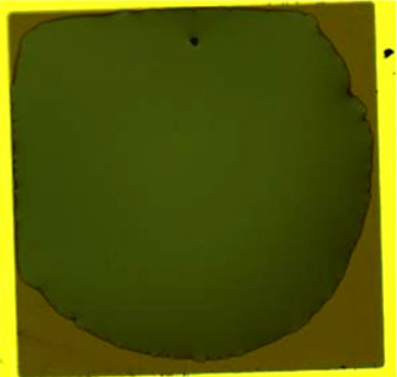

(c)

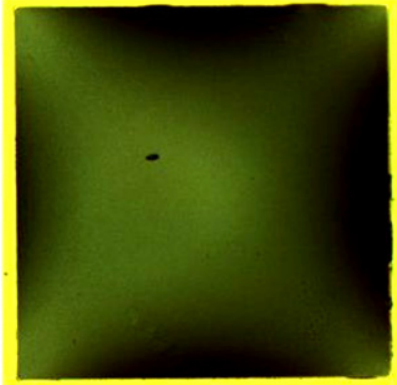

(b)

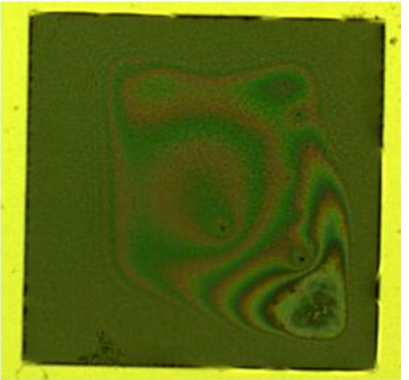

(d)
Figure 8. Water lenses over square $\mathrm{SiO}_{2} / \mathrm{CH}_{3}$ sites as obtained by dip-coating. Corner wetting is incomplete for superficial sites $(a)$ while perfectly conformal for recessed sites $(b)$. Absence and presence of water pinning at the site edges corresponded to different evaporation patterns, starting from the lens edge $(c)$ and from the centre $(d)$, respectively. Colours and depth enhanced by DIC optical microscopy. All site sides are $1 \mathrm{~mm}$ long.

purpose, we fabricated and dip-coated six sets of 24 isosceles triangular sites of the first class. The triangles were designed with a wide variety of angles, ranging from $2.5^{\circ}$ to $175^{\circ}$, and all the triangles of the same set had the same, constant area of 1 , $0.81,0.64,0.49,0.36$ and $0.25 \mathrm{~mm}^{2}$, respectively. Figure $9(a)$ shows the result of HD coating on the set of superficial triangles of $0.81 \mathrm{~mm}^{2}$ area. As expected, the coverage was largely nonconformal. The HD was not pinned but only constrained by the edges of the binding sites; the site corners were de-wetted by the lubricant. In contrast, all sets of sites of the recessed types were perfectly covered (figure $9(b)$ shows the case of $0.36 \mathrm{~mm}^{2}$ area). The sidewall pinning enforced conformal wetting inside every angle. This clearly showed that, in the absence of a defined wetting boundary along the site perimeter, the full site-covering state of the lenses has higher energy than the energy-minimizing state, i.e. the natural tendency of the lenses to assume their energy-minimizing configuration (given a fixed volume) is in general not compatible with full site coverage. Given the wide range of site shapes that we tested, we assume that our method can possibly work for sites of arbitrary shapes.

\section{Finite element simulations}

To support our experimental observations, numerical simulations of $\mathrm{HD}$ lenses over the $\mathrm{CH}_{3} / \mathrm{SiO}_{2}$ types of square sites were performed. Focusing on the equilibrium shape

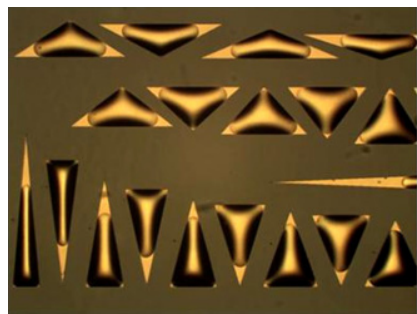

(a)

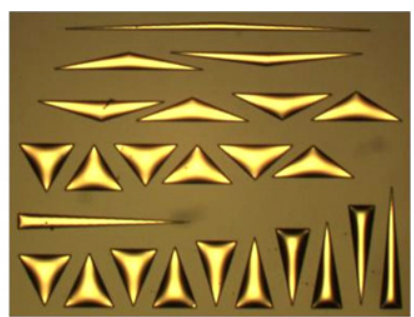

(b)
Figure 9. Hexadecane lenses on isosceles triangular $\mathrm{CH}_{3} / \mathrm{SiO}_{2}$ sites as obtained by dip-coating. The substrate is submerged in water. Sites designed to span a range of angles from $2.5^{\circ}$ to $175^{\circ}$, and areas from 1 to $0.25 \mathrm{~mm}^{2}$. Partial de-wetting from the site corners was always observed for superficial sites $((a)$, case for site area $=$ $0.81 \mathrm{~mm}^{2}$ ) as compared to perfectly conformal site coverage for all sites of the recessed type $\left((b)\right.$, case for site area $\left.=0.36 \mathrm{~mm}^{2}\right)$. Colours and depth enhanced by DIC optical microscopy.

(which constitutes a surface of constant mean curvature, as deriving from the Laplace equation [55]) of lubricant lenses as resulting from fluid deposition over binding sites, we opted for a quasi-static simulative approach using Surface Evolver (SE) [56] as simulation software. We thus excluded any consideration of dynamical (e.g. viscosity, coating speed, pinch-off) and inertial effects in the coating process. We were aware that this made the simulations not entirely realistic, since these effects do play an important role in the dip-coating performance. However, our approach mainly aimed at explicating the energetic underpinnings of the influence of the wetting sidewall and of other material parameters on the coverage results, an aim for which SE was specifically suited.

Square binding sites of the same dimensions $\left(1 \times 1 \mathrm{~mm}^{2}\right)$ as the ones used in experiments were simulated. Data for interfacial energies and fluid densities were taken from the literature [51].

\subsection{Site surface coverage}

First the impact of lubricant/binding site CA and of lubricant volume on the lens coverage over the superficial site type was studied. Figure 10 shows the site surface coverage as a function of the lubricant/site CA for a fixed lubricant volume of $65 \mathrm{~nL}$. The simulation confirmed that by decreasing the CA (thus increasing the wetting property of the lubricant by decreasing its interfacial tension) the lens contact area increased until the asymptotic filling of the entire site was achieved, which happened in the limit case of null contact angle (complete wetting). Full site coverage was also asymptotically obtained in the limit of large lubricant volumes at constant lubricant CA (fixed at $22^{\circ}$ ) (figure 11). In all the simulations, completely non-wetting boundary conditions at the edges of the binding site were introduced. This was consistent with experiments, as the $\mathrm{CA}$ of $\mathrm{HD}$ on silica in water is $180^{\circ}$. Any chemical and/or topological heterogeneity therefore produces complete confinement of the lubricant inside the site. 


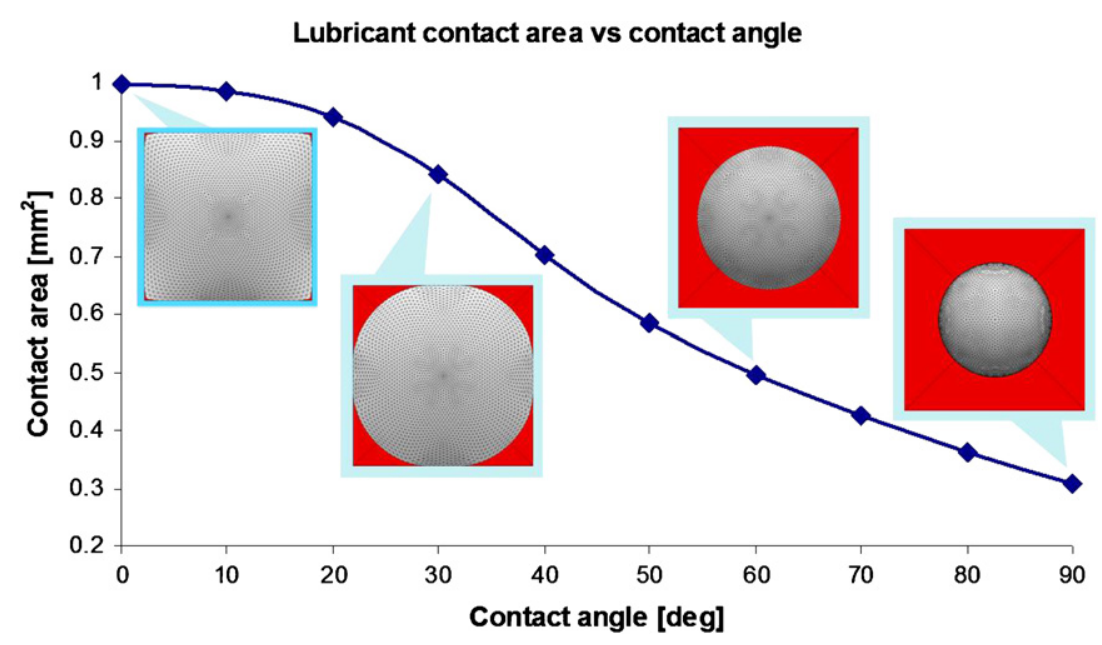

Figure 10. Lubricant contact area as a function of the lubricant/site contact angle on the $1 \mathrm{~mm}^{2}$ superficial square $\mathrm{CH}_{3} / \mathrm{SiO}_{2}$ site, after $\mathrm{SE}$ simulations. Fixed lubricant volume was $65 \mathrm{~nL}$. Insets show the site surface coverage by the lubricant lens. Full site coverage is asymptotically achieved in the limit of null contact angle (complete wetting).

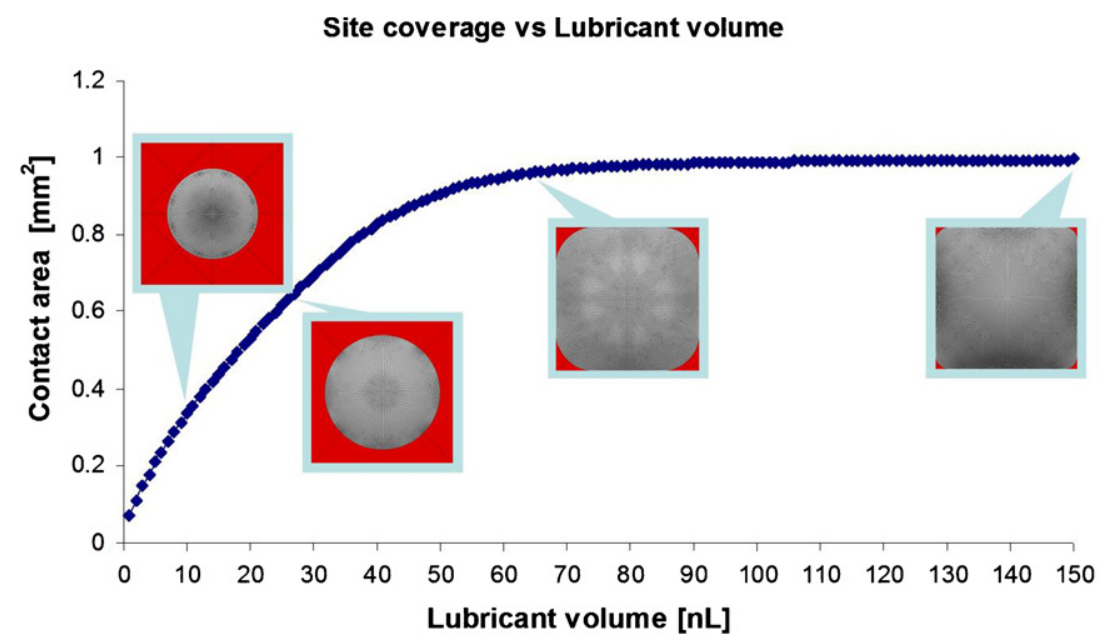

Figure 11. Lubricant contact area as a function of the lubricant volume on the $1 \mathrm{~mm}^{2}$ superficial square $\mathrm{CH}_{3} / \mathrm{SiO}_{2}$ site, after $\mathrm{SE}$ simulations. Fixed contact angle was $22^{\circ}$. For lubricant volumes larger than around $70 \mathrm{~nL}$ site coverage is almost constant. Full site coverage is asymptotically achieved in the limit of large lubricant volume.

\subsection{Energy-minimizing lubricant volumes}

The quasi-static equilibrium shape of HD lenses on both superficial and recessed types of square sites was also investigated. Specifically, the total energy of the system in its minimal energy state was calculated as a function of the volume of the lubricant lenses (a type of simulation first introduced by Srinivasan [51]). The total system energy included the gravitational energy of the lubricant and the interfacial energies associated with all the interfaces in the system. As a reference system, the lubricant coverage behaviour on a $1 \mathrm{~mm}^{2}$ circular superficial binding site was initially studied. The simulated energy function is depicted in figure 12. As the lubricant volume was increased, the system energy decreased because the highly energetic $\mathrm{SAM} /$ water interface was progressively substituted by the less energetic SAM/lubricant one, while the increasing gravitational contribution from the lubricant was negligible and that of the lubricant/water interface was nearly constant. The curve had a definite minimum which coincides with the value of lubricant volume $(53 \mathrm{~nL})$ for which complete site coverage was first achieved. After that, the system energy increased indefinitely because of the expansion of the lubricant/water interface while the SAM/lubricant interface was fixed. The cusp around the energy minimum is the signature of complete lubricant pinning due to the site edges. In comparison, the function simulated for the square superficial site (figure 13), while having an analogous overall trend, showed a less definite, broader energy minimum. We suppose this is due to the absence of lubricant pinning in the corners of the sites. This suggests that, in the absence of a wetting contour, hydrocarbon spreading inside the straight 


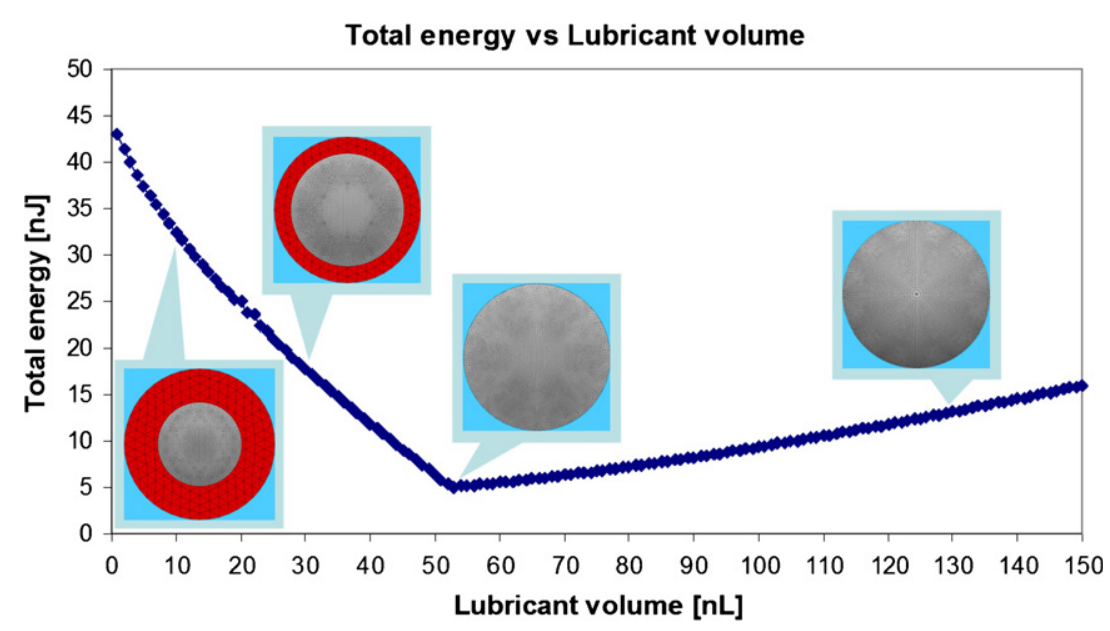

Figure 12. Total system energy as a function of the lubricant volume (constant contact angle $=22^{\circ}$ ) for the $1 \mathrm{~mm}^{2}$ circular superficial $\mathrm{CH}_{3} / \mathrm{SiO}_{2}$ site, after SE simulations. Minimal energy configuration achieved for a volume of $53 \mathrm{~nL}$. The cusp around the minimum is the signature for lubricant pinning.

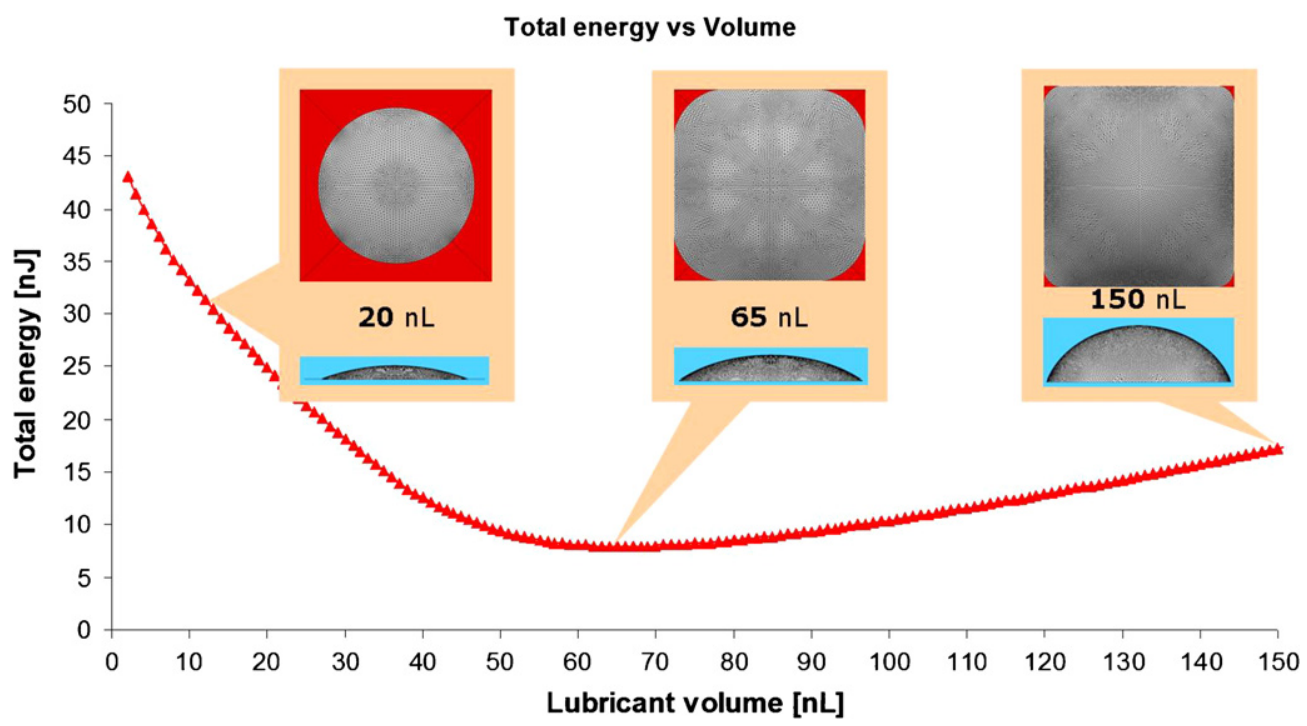

Figure 13. Total system energy as a function of the lubricant volume (constant contact angle $=22^{\circ}$ ) for the $1 \mathrm{~mm}^{2}$ square superficial $\mathrm{CH}_{3} / \mathrm{SiO}_{2}$ site, after SE simulations. Insets show the site surface coverage by the lubricant lens. Minimal energy configuration achieved for a lens volume of $65 \mathrm{~nL}$, for which the surface coverage is not complete. The broad energy minimum is related to the absence of pinning of the triple contact lines in the site corners.

angle edges of planar sites in water is energetically not favourable.

Simulations also showed that the ratio of lens contact area to superficial site area stayed constant as the side length of the site was scaled down: corner de-wetting appears to be scale-independent.

Using the same parameters as above for the simulation of a HD lens on a square site recessed into a $2.5 \mu \mathrm{m}$ deep cavity led to the results shown in figure 14 . The energy curve had again a definite minimum at the smallest lubricant volume $(40 \mathrm{~nL})$ that achieved full site coverage. The simulation dynamics clearly confirmed the role played by the wetting boundary for this type of site. The hydrocarbon lens preferentially spread along the sidewalls, and eventually achieved complete coverage of the site reaching into the corners. Only by wetting the hydrophobic sidewalls did the HD lens gain enough energy to balance the energetic costs of the local increase of surface curvature required to fill the square corners.

Notably, the numerically predicted equilibrium shape for lubricant lenses of minimal total energy closely reproduced experimental observations after dip-coating (compare e.g. the insets in figures $10-14$ to figures 5-8, and see figure 15 for the case of superficial and recessed equilateral triangular sites). 


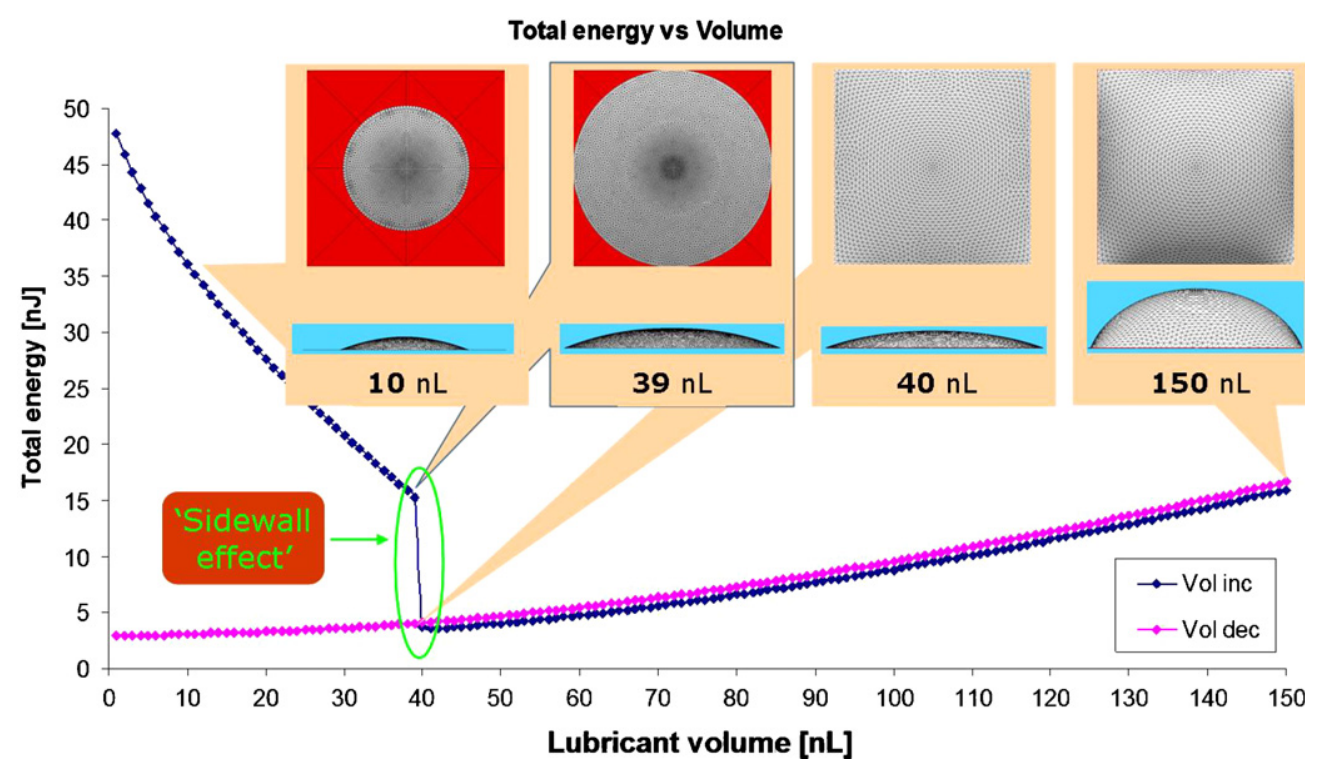

Figure 14. Total system energy as a function of the increasing lubricant volume (constant contact angle $=22^{\circ}$ ) for the $1 \mathrm{~mm}^{2}$ recessed $\mathrm{CH}_{3} / \mathrm{SiO}_{2}$ square site, after SE simulations. Insets show the site surface coverage by the lubricant lens. Minimal energy configuration achieved for a lens volume of $40 \mathrm{~nL}$, for which the surface coverage is complete thanks to sidewall pinning. By running the simulation with decreasing lubricant volume, no corner de-wetting is seen.

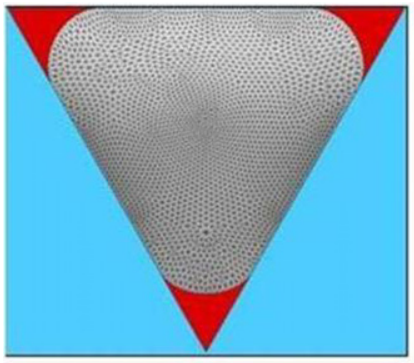

(a)

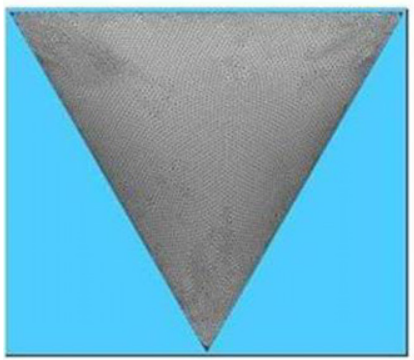

(c)

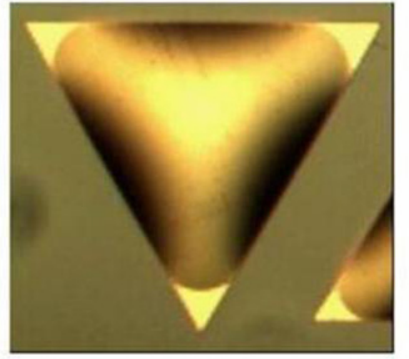

(b)

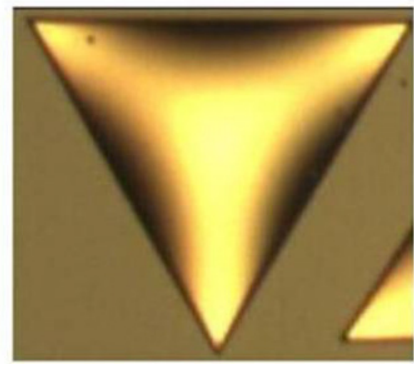

(d)

Figure 15. Comparison between simulated and experimental coating of equilateral triangular sites: $(a)$ and $(c)$ simulated minimal energy configuration for a lubricant lens on a superficial and recessed site, respectively; $(b)$ and $(d)$ hexadecane lens over a superficial and recessed site, respectively, after dip-coating. The substrate is submerged in water. Colour and depth enhanced by DIC optical microscopy. All sites are of the $\mathrm{CH}_{3} / \mathrm{SiO}_{2}$ class.

\section{Conclusions}

One of the key parameters for the yield of capillary die-tosubstrate self-assembly is the conformality of the lubricant lenses on binding sites patterned on planar substrates. In this paper we proposed a simple and rather general method to obtain conformal wetting of patterned sites based on the introduction of a wetting sidewall surrounding the entire site perimeter. We presented experimental and numerical evidence of the benefits brought by such topological site modification, including perfect conformality in the fluid coverage of arbitrarily shaped sites, superior coverage robustness and insensitivity against surface defects, and independence from the fluids and materials used, provided that a sufficiently large wetting contrast between the hydrophilic and hydrophobic regions is introduced. Notably, these advantages derived from a very small topological modification of the sites, since the 
thickness of the sidewall we used was only a negligible fraction of the side length of the sites.

Application of the surface preparation technique proposed here to the conformal fluid coverage of sites with arbitrary patterns (involving very acute angles and concavities, as well) could result in higher precision in die-to-substrate registration and larger freedom in site shape design. This is especially important for the purpose of achieving unique, arbitrary inplane placement and orientation of devices. Furthermore, the enhancement of the coating process reproducibility and insensitivity to surface defects brought by the proposed technique may significantly ease the adaptation of capillary die self-assembly to large substrates (e.g. $8^{\prime \prime}$ wafers).

While this work focused on blank substrates that were not pre-processed, a remaining challenge for the industrial success of capillary self-assembly is how to chemically and/or topologically condition back-end-of-line-processed substrates for the capillary process. This is necessary to accomplish the heterogeneous self-assembly of components on substrates containing electronics, interconnections, MEMS or other structures. Ideally, such conditioning should be compatible with all previous processing steps, and should not impose many and/or costly additional steps to the process flow. The definition of recessed binding sites would require, at the design level, an appropriate floorplanning of the target substrate to allow for their accurate location. The related, additional substrate processing would include, in the general case, thin film deposition, patterning, etching and functionalization. On the die side, matching patterns would need to be defined with similar operations. While their full impact on integration has not been completely investigated yet, it is the authors' opinion that these finalizing steps would be compatible with existing production flows and new approaches under development (e.g. 3D stacked ICs). Moreover, the added benefits of recessed binding sites, discussed above, may well repay the additional processing load, particularly in the perspective of assembling very large numbers of small components onto large preprocessed substrates.

\section{Acknowledgments}

The authors thank Ann Witvrouw, Eric Beyne and Paresh Limaye (Imec, Leuven, BE), Karl F Böhringer (University of Washington, Seattle, WA, USA) and Rajashree Baskaran (Intel, Seattle, WA, USA) for insightful discussions.

\section{References}

[1] Whitesides G M and Grzybowski B 2002 Self-assembly at all scales Science 295 2418-21

[2] Whitesides G M and Boncheva M 2002 Beyond molecules: self-assembly of mesoscopic and macroscopic components Proc. Natl Acad. Sci. 99 4769-74

[3] Boncheva M, Bruzewicz D A and Whitesides G M 2003 Millimeter-scale self-assembly and its applications Pure Appl. Chem. 75 621-30

[4] Goodman L S 1969 Geometrical optimization of controlled collapse interconnections IBM J. Res. Dev. 13 251-65
[5] Van Brussel H, Peirs J, Reynaerts D, Delchambre A, Reinhart G, Roth N, Weck M and Zussman E 2000 Assembly of microsystems Ann. CIRP 49 451-72

[6] Cohn M B, Bohringer K F, Noworolski J M, Singh A, Keller C G, Goldberg K Y and Howe R T 1998 Microassembly technologies for MEMS SPIE Micromachining and Microfabrication Process Technology IV

[7] Fearing R S 1995 Survey of sticking effects for micro parts handling IEEE/RSJ Int. Workshop on Intelligent Robots and Systems (IROS)

[8] Arai F, Ando D and Fukuda T 1996 Adhesion forces reduction for micromanipulation based on micro physics IEEE Micro Electro Mechanical Systems 1996

[9] Yeh H-J J and Smith J S 1994 Fluidic assembly for the integration of GaAs light-emitting diodes on Si substrates IEEE Photonics Technol. Lett. 46 706-9

[10] Verma A K, Hadley M A, Yeh H-J J and Smith J S 1995 Fluidic self-assembly of silicon microstructures IEEE Electronic Components and Technology Conf.

[11] Smith J S 2000 High density, low parasitic direct integration by fluidic self assembly (FSA) Int. Electron Devices Meeting 2000

[12] Yeh H-J J and Smith J S 1994 Fluidic self-assembly of microstructures and its application to the integration of GaAs on Si IEEE Workshop on Micro Electro Mechanical Systems (MEMS 1994)

[13] Edman C F, Swint R B, Gurtner C, Formosa R E, Roh S D, Lee K E, Swanson P D, Ackley D E, Coleman J J and Heller M J 2000 Electric field directed assembly of an InGaAs LED onto silicon circuitry IEEE Photonics Technol. Lett. 12 1198-200

[14] Lee S W, McNally H A, Guo D, Pingle M, Bergstrom D E and Bashir R 2002 Electric-field-mediated assembly of silicon islands coated with charged molecules Langmuir $183383-6$

[15] O'Riordan A, Delaney P and Redmond G 2004 Field configured assembly: programmed manipulation and self-assembly at the mesoscale Nanoletters 4 761-5

[16] Lee S W and Bashir R 2005 Dielectrophoresis and chemically mediated directed self-assembly of micrometer-scale three-terminal metal oxide semiconductor field-effect transistor Adv. Mater. 17 2671-7

[17] Yellen B B and Friedman G 2004 Programmable assembly of heterogeneous colloidal particle arrays Adv. Mater. 16 111-5

[18] Ramadan Q, Uk Y S and Vaidyanathan K 2007 Large scale microcomponents assembly using external magnetic array Appl. Phys. Lett. 90172502

[19] Shetye S B, Eskinazi I and Arnold D P 2008 Self-assembly of millimeter-scale components using integrated micromagnets IEEE Trans. Magn. 44 4293-6

[20] Zheng W, Chung J and Jacobs H O 2006 Fluidic heterogeneous microsystems assembly and packaging IEEE J. MEMS 15 864-70

[21] Chung J, Zheng W, Hatch T J and Jacobs H O 2006 Programmable reconfigurable self-assembly: parallel heterogeneous integration of chip-scale components on planar and nonplanar surfaces IEEE J. MEMS 15 457-64

[22] Stauth S A and Parviz B A 2006 Self-assembled single-crystal silicon circuits on plastic Proc. Natl Acad. Sci. $10313922-7$

[23] Srinivasan U, Liepmann D and Howe R T 2001 Microstructure to substrate self-assembly using capillary forces IEEE $J$. MEMS 10 17-24

[24] Xiong X, Hanein Y, Fang J, Wang Y, Wang W, Schwartz D T and Bohringer K F 2003 Controlled multibatch self-assembly of microdevices IEEE J. MEMS 12 117-27 
[25] Tu J K, Talghader J J, Hadley M A and Smith J S 1995 Fluidic self-assembly of InGaAs vertical cavity surface emitting lasers onto silicon IEEE Electron. Lett. 31 1448-9

[26] Gracias D H, Tien J, Breen T L, Hsu C and Whitesides G M 2000 Forming electrical networks in three dimensions by self-assembly Science $\mathbf{2 8 9}$ 1170-2

[27] Jacobs H O, Tao A R, Schwartz A, Gracias D H and Whitesides G M 2002 Fabrication of a cylindrical display by patterned assembly Science $296323-5$

[28] Srinivasan U, Helmbrecht M A, Rembe C, Muller R S and Howe R T 2002 Fluidic self-assembly of micromirrors onto microactuators using capillary forces IEEE J. Sel. Top. Quantum Electron. 8 4-11

[29] Lee S W and Bashir R 2003 Dielectrophoresis and electrohydrodynamics-mediated fluidic assembly of silicon resistors Appl. Phys. Lett. 83 3833-5

[30] Scott K, Hirano T, Yang H, Singh H, Howe R T and Niknejad A M 2004 High-performance inductors using capillary based fluidic self assembly IEEE J. MEMS 13 300-9

[31] Fang J, Wang K and Bohringer K F 2006 Self-assembly of PZT actuators for micropumps with high process repeatability IEEE J. MEMS 15 871-8

[32] Soga I, Hayashi S, Ohno Y, Kishimoto S, Maezawa K and Mizutani T 2005 Direct integration of GaAs HEMTs on AlN ceramic substrates using fluidic self-assembly IEEE Electron. Lett. 41 1275-6

[33] Terfort A, Bowden N and Whitesides G M 1997 Three-dimensional self-assembly of millimetre-scale components Nature 386 162-4

[34] Schunk P R, Hurd A J and Brinker C J 1997 Free-meniscus coating processes Liquid Film Coating ed S F Kistler and P M Schweizer (London: Chapman and Hall)

[35] Landau L and Levich B 1942 Dragging of a liquid by a moving plate Acta Physicochim. URSS 1742

[36] Deryagin B M and Levi S M 1964 Film Coating Theory (New York: Focal Press)

[37] Darhuber A A, Troian S M, Davis J M and Miller S M 2000 Selective dip-coating of chemically micropatterned surfaces J. Appl. Phys. 88 5119-26

[38] Hartmann D M, Kibar O and Esener S C 2001 Optimization and theoretical modeling of polymer microlens arrays fabricated with the hydrophobic effect Appl. Opt. $402736-46$

[39] Jackmann R J, Duffy D C, Ostuni E, Willmore N D and Whitesides G M 1998 Fabricating large arrays of microwells with arbitrary dimensions and filling them using discontinuous dewetting Anal. Chem. 70 2280-7

[40] Meyer E E, Rosenberg K J and Israelachvili J 2006 Recent progress in understanding hydrophobic interactions Proc. Natl Acad. Sci. 103 15739-46
[41] Liang S-H, Wang K and Bohringer K F 2005 Self-assembly of MEMS components in air assisted by diaphragm agitation IEEE Int. Conf. on MEMS 2005

[42] Sato K, Lee K, Nishimura M and Okutsu K 2007 Self-alignment and bonding of microparts using adhesive droplets Int. J. Precis. Eng. Manuf. 8 75-9

[43] Bohringer K F, Srinivasan U and Howe R T 2001 Modelling of capillary forces and binding sites for fluidic self-assembly IEEE Int. Conf. on MEMS

[44] Xiong X, Liang S-H and Bohringer K F 2004 Geometric binding site design for surface-tension driven self-assembly IEEE Int. Conf. on Robotics and Automation (ICRA) (New Orleans, 2004)

[45] Liang S-H, Xiong X and Bohringer K F 2004 Toward optimal designs for self-alignment in surface-tension driven micro-assembly IEEE Conf. on MEMS (Maastricht, 2004)

[46] Sato K, Ito K, Hata S and Shimokohbe A 2003 Self-alignment of microparts using liquid surface tension-behaviour of micropart and alignment characteristics Precis. Eng. 27 42-50

[47] Biebuyck H A and Whitesides G M 1994 Self-organization of organic liquids on patterned self-assembled monolayers of alkanethiolates on gold Langmuir 10 2790-3

[48] Greiner A, Lienemann J, Kornink J G, Xiong X, Hanein Y and Bohringer K F 2002 Capillary forces in micro-fluidic self-assembly 5 th Int. Conf. on Modeling and Simulation of Microsystems (MSM'02) (San Juan, 2002)

[49] Lieneman J, Greiner A, Kornink J G, Xiong X, Hanein Y and Bohringer K F 2003 Modelling, simulation and experiment of a promising new packaging technology - parallel fluidic self-assembly of microdevices Sens. Update 13 3-43

[50] Abbott N L, Whitesides G M, Racz M L and Szekely J 1994 Using finite element analysis to calculate the shapes of geometrically confined drops of liquid on patterned, self-assembled monolayers: a new method to estimate excess interfacial free energies J. Am. Chem. Soc. 116 290-4

[51] Srinivasan U 2001 Fluidic self-assembly of microfabricated parts to substrates using capillary forces $P h D$ Thesis University of California at Berkeley

[52] Love J C, Estroff L A, Kriebel J K, Nuzzo R G and Whitesides G M 2005 Self-assembled monolayers of thiolates on metals as a form of nanotechnology Chem. Rev. 105 1103-69

[53] Houston M R and Maboudian R 1995 Stability of ammonium fluoride-treated $\mathrm{Si}(100) \mathrm{J}$. Appl. Phys. 78 3801-8

[54] Erbil H Y, McHale G and Newton M I 2002 Drop evaporation on solid surfaces: constant contact angle mode Langmuir 18 2636-41

[55] Gau H, Herminghaus S, Lenz P and Lipowsky R 1999 Liquid morphologies on structured surfaces: from microchannels to microchips Science $\mathbf{2 8 3} 46-9$

[56] Brakke K A 1992 The surface evolver Exp. Math. 1 141-65 